\title{
Recurrent Myxoid Liposarcoma
}

National Cancer Institute

\section{Source}

National Cancer Institute. Recurrent Myxoid Liposarcoma. NCI Thesaurus. Code C150579.

The reemergence of a myxoid liposarcoma after a period of remission. 